\title{
Wertvolles Instrument
} statt lästige Pflicht

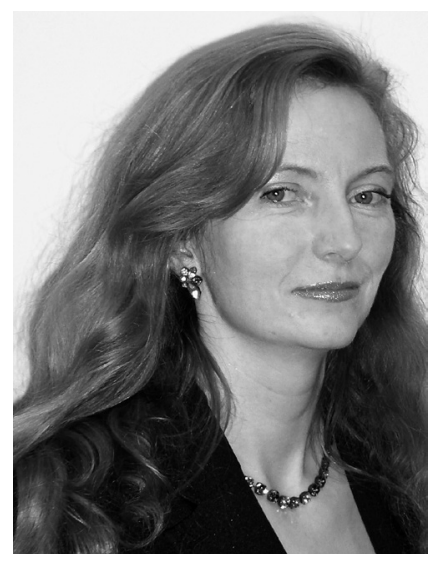

VON DIETGARD SALEIN Dietgard Salein arbeitet als selbstständige Personalberaterin in Berlin. Neben ihren Tätigkeitsschwerpunkten Bewerbungsberatung und Karrierecoaching gibt sie Seminare zu Themen wie Konfliktmanagement und Beurteilungswesen.

www.personal-salein.de

\author{
Das oft als lästig empfundene Mitarbeitergespräch \\ kann ein wertvolles Personalinstrument sein, in \\ dem es neben der Beurteilung immer auch um \\ Orientierung und Förderung gehen sollte.
}

Es nennt sich Jahresgespräch, Mitarbeitergespräch oder einfach Beurteilungsgespräch - nicht zu erwähnen die vielen verschiedenen hausinternen Bezeichnungen der Unternehmen, die dieses Instrument nutzen. Gemeint ist damit jedoch immer eines: die wertschätzende Beurteilung der Arbeitsleistung einer Mitarbeiterin oder eines Mitarbeiters, verbunden mit individueller Zielsetzung und Fördervereinbarung. So sollte es zumindest sein.

Und damit stellt das Mitarbeitergespräch - einigen wir uns auf diesen Begriff - eines der besten und effizientesten Führungsinstrumente dar, die modernen Unternehmen zur Verfügung stehen. Seine Vorteile für Mitarbeiter, Vorgesetzte und Unternehmen sind vielfältig:

- Der Mitarbeiter erhält eine verlässliche Rückmeldung, ob und wie zufrieden man mit ihm und seiner Leistung ist.

- Der Vorgesetzte erfährt etwas über die Erwartungen, die der Mitarbeiter an ihn hat, und bekommt vielleicht ein Feedback über seine Leistung als Chef.

Gerade, wenn das normale Tagesgeschäft wenig Zeit für den regelmäßigen Austausch zwischen Vorgesetzten und Mitarbeitern lässt, bietet das Mitarbeitergespräch die Möglichkeit, den persönlichen Kontakt zwischen beiden Ebenen zu vertiefen. Das wirkt motivierend, erhöht die Zufriedenheit der Mitarbeiter und verbessert damit die Arbeitsleistung.

\section{Verschleißerscheinungen erkennen}

Hört man sich jedoch um, so ist das Mitarbeitergespräch weder bei Vorgesetzten noch bei Mitarbeitern allzu beliebt; es wird eher als lästig bis verhasst eingestuft. Was oft mit viel Aufwand erdacht und zunächst mit Eifer umgesetzt wurde, nämlich ein teures, genau auf die Belange eines Unternehmens abgestimmtes System - mit individuell ausgearbeiteten Formularen, Schulungsreihen für Führungskräfte, einem Leitfaden im Ausmaß eines Buches -, zeigt schon nach wenigen Jahren des Gebrauchs erste Verschleißerscheinungen.

Da ist zum einen das Alltagsgeschäft, das scheinbar keine Zeit lässt für allzu ausführliche Gespräche - und schon gar nicht jedes Jahr und mit jedem Mitarbeiter! Unter der Hand fallen dann schon mal Sätze wie: »Was, Frau Mustermann, Sie wollen eine Beurteilung? Sehen sie mal, ich bin doch so zufrieden mit Ihnen, das müsste Ihnen doch reichen!« Welcher Mitarbeiter wird dann schon auf seinem Recht beharren? Gern wird auch mal die Beurteilung vom Vorjahr übernommen und mit einem neuen $\mathrm{Da}$ tum versehen.

Leider wird das Mitarbeitergespräch aber oft auch dazu benutzt, dem Mitarbeiter all das zu sagen, was einen schon lange geärgert hat und ihn mit all den Verfehlungen zu konfrontieren, die er übers Jahr begangen hat und wozu man sonst nicht kam - oder nicht kommen wollte. Am Tag der Beurteilung rechnet der Mitarbeiter doch sowieso mit Kritik, dann ist das »ein Abwasch« ... 
Aber genau dadurch kommt das Mitarbeitergespräch in Verruf, schlechte Erfahrungen sprechen sich herum, ein offenes Gespräch über Vorstellungen und Ziele ist nicht mehr ohne weiteres möglich und auf Jahre sorgt die bloße Androhung des Gesprächs für erhöhten Puls.

Daher gilt als erste Regel: Das Mitarbeitergespräch dient nicht der Abstrafung! Tadel oder gar Abmahnung für konkrete Verfehlungen gehören da nicht hinein! So etwas klärt ein guter Vorgesetzter direkt und zeitnah. Und das Ziel ist dabei immer eine Verbesserung der Arbeitsleistung, nicht die Hinrichtung des Mitarbeiters!

Investieren Sie Zeit und Aufmerksamkeit, und zwar sowohl in die Vorbereitung, als auch in die Durchführung! Wird das Mitarbeitergespräch schlecht vorbereitet oder sozusagen im Vorbeigehen abgehandelt, richtet es mehr Schaden an, als wenn es gar nicht stattfindet. Der Mitarbeiter merkt, wenn ein Chef sich nicht sorgfältig vorbereitet hat. Die Beurteilung, selbst wenn sie gut ausfällt, wird dann nicht wirklich ernst genommen und der Chef selbst verliert an Respekt bei seinen Mitarbeitern.

Mit dem Respekt ist das überhaupt so eine Sache: Als Vorgesetzter müssen Sie in Vorleistung gehen! Begegnen Sie dem Mitarbeiter mit Respekt und Wertschätzung, können Sie Entsprechendes vom Mitarbeiter erwarten. Das gilt selbstverständlich auch im Mitarbeitergespräch. Überlegen Sie sich, was Sie darin eigentlich mitteilen wollen und formulieren Sie vor! Fragen Sie ruhig einen Dritten um Rat, ob das, was Sie sagen wollen, auch »rüberkommt «. Nehmen Sie aber unbedingt jemanden, der den Mitarbeiter nicht kennt, aber dafür Sie, ihre Stärken und Ihre Schwächen auf dem Gebiet der Kommunikation.

Natürlich gehört Kritik ins Mitarbeitergespräch, wenn sie angebracht ist und konstruktiv geäußert wird. Sparen Sie nicht mit Lob, denn nichts wirkt so motivierend, und nebenbei ist es auch noch preiswert! Beides - Kritik wie Lob - muss nachvollziehbar begründet und berechtigt sein, aber dass es nichts zu loben gibt, kommt eher nicht vor, es sei denn, Sie oder Ihr Unternehmen hätten sich bei der Personalauswahl komplett vergriffen. Und in diesem Falle wäre zu fragen, wer eigentlich kritisiert werden muss ...
Damit aus einer bloßen Beurteilung ein Mitarbeitergespräch wird, gehört die Absprache der künftigen Aufgabengestaltung und ein offenes Gespräch über die beruflichen und persönlichen Zielvorstellungen des Mitarbeiters dazu. Fragen Sie ihn, wie er sich weiterentwickeln möchte! Tun Sie es nicht, werden es andere tun, verlassen Sie sich darauf!

Und keine Sorge, Förderung hat nicht unbedingt etwas mit Geldausgeben zu tun und es geht auch nicht immer um Be-Förderung. Überlegen Sie sich gemeinsam mit Ihrem Mitarbeiter eine herausfordernde Aufgabe für das kommende Jahr! Es muss nicht gleich die zehnprozentige Umsatzsteigerung sein. Die Herausforderung muss zum Mitarbeiter passen. Sie kann im Ausprobieren eines neuen PC-Programmes bestehen, das anschließend der Abteilung vorgestellt und auf seine Brauchbarkeit hin bewertet wird oder darin, sich ein neues Ablagesystem zu überlegen. Oder schicken Sie Ihren Mitarbeiter für eine Woche in eine andere Abteilung, damit er sieht, wo seine Zuarbeit landet! Im Zweifel haben Ihre Mitarbeiter auch Ideen, die sie gerne ausprobieren wollen, sich aber nicht trauen oder die sie selbst nicht für so bedeutend erachten.

\section{Reflexion}

Alles, was Sie im Mitarbeitergespräch besprechen, muss schriftlich fixiert werden, nicht nur die Beurteilung, sondern auch die Aufgabenzuteilung und jede Fördervereinbarung; nur dann wird sie von beiden Teilnehmern als bindend anerkannt. Wichtig ist, dass Sie die vereinbarten Maßnahmen in der abgesprochenen Zeit veranlassen und überprüfen. Das dient nicht nur der Kontrolle, sondern auch Ihrem guten Ruf.

Führen Sie das Mitarbeitergespräch einmal im Jahr mit jedem Mitarbeiter, so bleiben Sie in Kontakt und können beim nächsten Mal noch auf die letzte Unterredung aufbauen. Der Lohn für Ihre Kontinuität ist ein stabiles Vertrauensverhältnis zu Ihren Mitarbeitern, von dem letztlich Sie profitieren.

All das ist nichts Neues. Wir haben die entsprechenden Schulungen für Mitarbeitergespräche besucht, wir haben über die Jahre Erfahrungen gesammelt und wir wissen auch über Lob und Respekt Bescheid. Und trotzdem ist es angebracht, den alten Beurteilungsleitfaden hin und wieder zur Hand zu nehmen und aufmerksam durchzulesen und sich kritisch zu fragen, ob wir wirklich auf alles achten, was wichtig ist. Das eine oder andere ist oft gerade dem Routinierten abhandengekommen, sei es aus Gewohnheit oder Bequemlichkeit. Schon eine Checkliste, die man vor dem Gespräch noch einmal durchliest, kann sehr hilfreich sein!

Und so wie auch die Werkzeuge in einer Werkstatt von Zeit zu Zeit einer Prüfung und Generalüberholung bedürfen, damit sie verlässlich ihren Dienst tun, lohnt es sich auch, ein bewährtes Führungsinstrument wie das hauseigene Beurteilungssystem regelmäßig zu hinterfragen, zeitgemäß zu halten und zu pflegen. Nur dann bleibt es, was es sein soll: keine lästige Pflicht, sondern eines der besten und effizientesten Führungsinstrumente, die modernen Unternehmen zur Verfügung stehen.

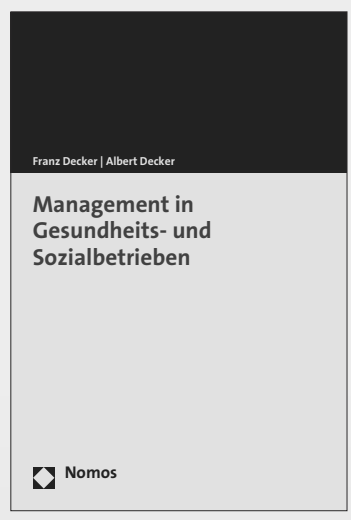

Management in Gesundheits- und Sozialbetrieben

Betriebswirtschaftliche Grundlagen für Führungskräfte und Nachwuchs Von Prof. Dr. Franz Decker und Dr. Albert Decker

2. Auflage 2008, 432 S., geb., 74,- $€$, ISBN 978-3-8329-2990-9

Portofreie Buch-Bestellungen unter www.nomos-shop.de/9500

Nomos 